\title{
Surface Sensing of Quantum Dots by Electron Spins
}

\author{
Fabrizio Moro, ${ }^{* \dagger}$ Lyudmila Turyanska, ${ }^{\dagger,}$ James Wilman, ${ }^{\dagger}$ Huw E. L. Williams, ${ }^{\S}$ Alistair J. Fielding, ${ }^{\|}$
} and Amalia Patanè

\author{
${ }^{\dagger}$ School of Physics and Astronomy, The University of Nottingham, Nottingham NG7 2RD, United Kingdom \\ ${ }^{\sharp}$ School of Chemistry, University of Lincoln, Lincoln LN6 7DL, United Kingdom \\ ${ }^{\S}$ Centre for Biomolecular Sciences, School of Chemistry, The University of Nottingham, Nottingham NG7 2RD, United Kingdom \\ "The Photon Science Institute and School of Chemistry, University of Manchester, Manchester M13 9PL, United Kingdom
}

\section{Supporting Information}

ABSTRACT: The nanoscale design of quantum dots (QDs) requires advanced analytical techniques. However, those that are commonly used do not have sufficient sensitivity or spatial resolution. Here, we use magnetic resonance techniques combined with paramagnetic $\mathrm{Mn}$ impurities in $\mathrm{PbS}$ QDs for sensitive probing of the QD surface and environment. In particular, we reveal inequivalent proton spin relaxations of the capping ligands and solvent molecules, strengths and anisotropies of the $\mathrm{Mn}$ nuclear spin interactions, and $\mathrm{Mn}$ nuclei distances with $\sim 1 \AA$ sensitivity. These findings demonstrate the potential of magnetically doped QDs as sensitive magnetic nanoprobes and the use of electron spins for surface sensing.

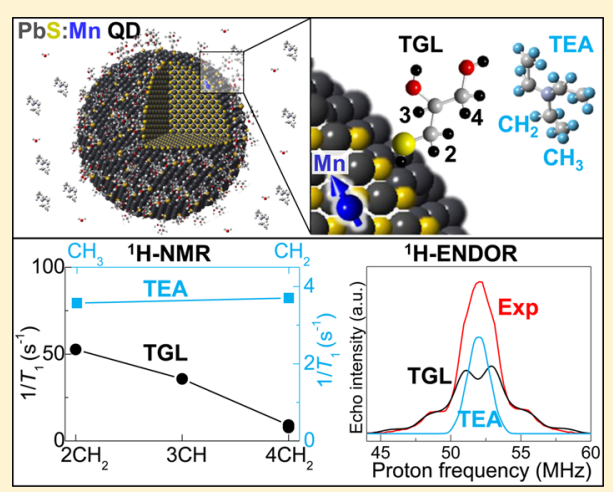

KEYWORDS: Colloidal quantum dots, magnetic doping, nuclear magnetic resonance (NMR), electron spin resonance (ESR), electron spin echo envelope modulation (ESEEM), electron-nuclear double resonance (ENDOR)

$\mathrm{N}$ anoscale design of colloidal quantum dots (QDs) by the controlled incorporation of paramagnetic impurities or modification of the surface by capping ligands requires analytical techniques with sufficient sensitivity to resolve single atoms and to probe surface properties. Both the position of the impurities in the host crystal lattice and their interaction with the surrounding environment (e.g., capping ligands, solvent, etc.) are central to the design and exploitation of the nanocrystals in several applications spanning optoelectronics ${ }^{1-3}$ and medical imaging. For example, multimodal imaging could be enabled by doping QDs with paramagnetic centers. ${ }^{4}$

Among several spectroscopic techniques, nuclear magnetic resonance (NMR) and electron spin resonance (ESR) stand out as they can resolve the structure and functionalities of systems that lack long-range order (e.g., proteins and biological membranes), which are otherwise inaccessible with more conventional techniques, such as X-ray diffraction (XRD). To date, NMR has been employed to probe the structure and surfaces of colloidal metal nanoparticles ${ }^{5}$ and quantum dots (QDs). ${ }^{6-9}$ In particular, solid-state NMR has enabled studies of the incorporation of low concentration of impurities. ${ }^{10}$ However, sample preparation involves the precipitation of the nanoparticles, which can affect the nanocrystal surface. In contrast, solution proton-NMR $\left({ }^{1} \mathrm{H} \mathrm{NMR}\right)$ is a nondestructive technique ${ }^{5,8,11-13}$ and could be equally informative. Indeed, spectral broadening and proton spin relaxations studies have been used to investigate the interfacial electronic structure in colloidal QDs ${ }^{14}$ as well as surface charge transfer processes. ${ }^{15,16}$

ESR is less commonly used than NMR because it relies on the presence of unpaired electrons. This requirement can be fulfilled by doping the nanocrystals with paramagnetic centers. The detection of electron spin impurities by pulsed-ESR methods combined with theoretical modeling enables the determination of structural and dynamical properties with nanosecond resolution. ${ }^{17}$ Pulsed-ESR has now become a standard approach for the characterization of proteins using site-directed mutagenesis and spin labeling, ${ }^{18,19}$ nanocrystals doped with magnetic impurities ${ }^{20,21}$ or radiation defects, ${ }^{2,23}$ as well as a method for the detection of NV-centers in diamond for biomarkers, ${ }^{24}$ spin qubits, ${ }^{25}$ and nanoscale sensors. ${ }^{25,26}$

Here, we demonstrate that the combined use of ${ }^{1} \mathrm{H}$ NMR and pulsed-ESR in colloidal QDs containing paramagnetic impurities enables sensitive probing of the QDs surface and environment. We probe the location of $\mathrm{Mn}^{2+}$ impurities in colloidal $\mathrm{PbS} \mathrm{QDs}$ and show that the interaction of single impurities with near protons provides a tool for discrimination between inequivalent proton spin relaxations of surrounding molecules, i.e., capping ligands and solvent molecules. In addition, we determine the strength and anisotropies of the

Received: July 2, 2016

Revised: September 9, 2016

Published: September 14, 2016 
interactions between surface $\mathrm{Mn}$ electron spins and near nuclear spins, as well as the Mn nuclei distances with $\sim 1 \AA$ sensitivity. The proximity of the Mn ions to the surface of the QDs provides us with a nanostructure whose magnetic properties are strongly sensitive to its environment. On the one hand, these results provide insight on the sources of decoherence for electron spins in QDs relevant for their application as qubits. ${ }^{27,28}$ On the other hand, they are relevant for future exploitation of magnetically doped QDs as sensitive magnetic nanoprobes in medical imaging. ${ }^{4}$

Colloidal Mn-doped $\mathrm{PbS}$ nanocrystals were synthesized in aqueous solution with $\mathrm{Mn}$ content from $0.01 \%$ to $0.1 \%$ and stabilized with a mixture of 1-thioglycerol (TGL) and 2,3dimercapto-1-propanol (DTG). Triethylamine (TEA) was used to control the $\mathrm{pH}$ of the $\mathrm{QD}$ solution. The $\mathrm{Mn}^{2+}$ ions are incorporated into the $\mathrm{Pb}$-sublattice sites of the $\mathrm{PbS}$ rock-salt cubic crystal. ${ }^{29}$ High-resolution transmission electron microscopy (HRTEM) studies (Figure 1a) show that the nanocrystals
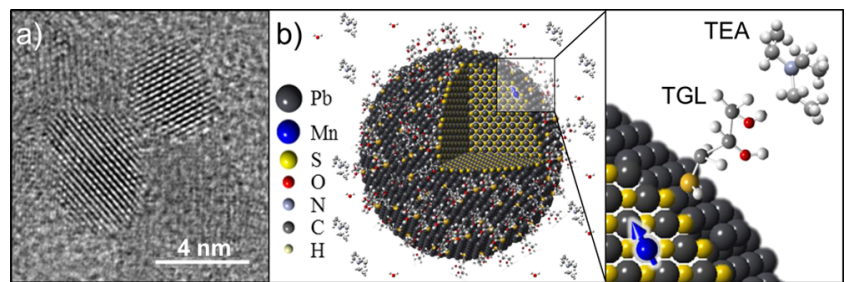

Figure 1. (a) High resolution TEM image of PbS:Mn QDs $(x=5 \%)$. (b) Schematic of a PbS:Mn QD and zoom of a surface $\mathrm{Mn}$ ion interacting with the TGL capping ligands and TEA solvent molecules in water solution.

retain high crystallinity and have an average diameter of $4.5 \pm$ $1.2 \mathrm{~nm}$. Our previous studies have demonstrated room temperature photoluminescence emission tunable by the $\mathrm{Mn}$ content in the range $950-1200 \mathrm{~nm} .^{29,30}$

The NMR data were collected at $600 \mathrm{MHz}$ on a Bruker Avance III spectrometer at $298 \mathrm{~K}$ on QDs dispersed in $\mathrm{H}_{2} \mathrm{O}: \mathrm{D}_{2} \mathrm{O}(1: 9 \mathrm{v} / \mathrm{v})$. Solvent suppression was achieved using excitation sculpting, where required. Spin-lattice relaxation time $\left(T_{1}\right)$ experiments were conducted with an inversion recovery sequence: $\operatorname{rf}(\pi)$ - delay $(\mathrm{T})-\operatorname{rf}(\pi / 2)-$ free induction decay, where $\mathrm{rf}$ stands for radiofrequency pulse duration and $\pi / 2=11.5 \mu \mathrm{s}$. Data were acquired as a pseudo $2 \mathrm{D}$ spectrum, and the relaxation delay was set at $>5 T_{1}$ to facilitate complete recovery between transients. Data were phased and baseline corrected prior to integration using TOPSPIN 3 software.

Pulsed-ESR studies were conducted on a PbS:Mn solid state sample with $\mathrm{Mn}$ concentrations of $0.03-0.05 \%\left(\sim 1 \mathrm{Mn}^{2+}\right.$ ion per QD), which provide a good compromise between long spin relaxation times $\left(T_{\mathrm{M}} \sim 1 \mu \mathrm{s}\right.$ and $T_{1} \sim 200 \mu \mathrm{s}$ at $\left.T=5 \mathrm{~K}\right)$ and a large signal/noise ratio in the pulsed-ESR experiments. ${ }^{31}$ Electron spin echo envelope modulation (ESEEM) and electron-nuclear double resonance (ENDOR) experiments were conducted on protonated and deuterated (D-PbS:Mn) solid state samples and performed at Q-band $\left(\nu_{\mathrm{mw}}=33.85\right.$ $\mathrm{GHz})$ at the fourth hyperfine peak $(B=1.211 \mathrm{~T})$ of an echo field swept spectrum (see Figure $S 1$ in the Supporting Information), which corresponds to the $m_{I}=-1 / 2$ nuclear line of the $m_{s}=+1 / 2 \leftrightarrow-1 / 2$ transition. ESEEM data were collected using a standard Hahn echo pulse sequence: $\pi / 2-$ delay $(\tau)-\pi-$ delay $(\tau)-$ echo signal, with $\pi=48$ ns and $\tau=$
200 ns. ENDOR data were collected using standard Davies: $\operatorname{mw}(\pi)$ - delay $(T)-\operatorname{rf}(\pi)-$ delay $(T)-\operatorname{mw}(\pi / 2)-$ delay $(\tau)-\operatorname{mw}(\pi)-$ delay $(\tau)$ - echo signal, where mw stands for microwave pulse duration, $\operatorname{rf}(\pi)=30 \mu$ s and $T=100 \mathrm{~ns}$; and Mims: $\operatorname{mw}(\pi / 2)-$ delay $(\tau)-\operatorname{mw}(\pi / 2)-$ delay $(T)-\operatorname{rf}(\pi)$ - delay $(T)-\operatorname{mw}(\pi / 2)$ - delay $(\tau)$ - echo signal pulse sequences, where $T=100 \mathrm{~ns}$. The experimental spectra were simulated using Easyspin software. ${ }^{32}$

Figure 1 shows a typical HRTEM image of Mn-doped PbS QDs and a schematic of a PbS QD with a Mn atom near its surface, a TGL ligand, which binds to the QDs via the SH group, and a TEA molecule. ${ }^{1} \mathrm{H}$ NMR spectra were acquired to probe the QD surface and environment and were compared to those of free TGL and TEA molecules.

The ${ }^{1} \mathrm{H}$ NMR spectrum of PbS QDs (Figure 2a) reveals two intense peaks centered at $\delta=1.24$ and $3.12 \mathrm{ppm}$, which we
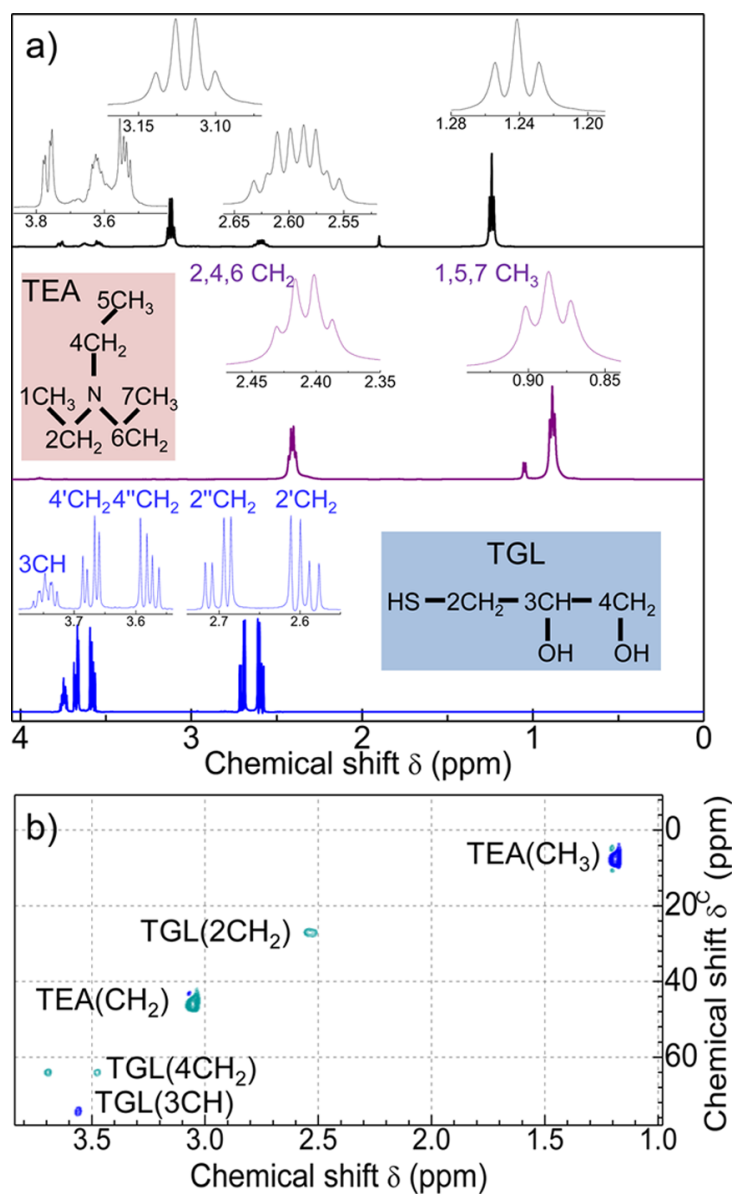

Figure 2. (a) ${ }^{1} \mathrm{H}$ NMR spectra for solutions of undoped PbS QDs (top panel), free TEA (middle panel), and free TGL molecules (bottom panel). Insets are skeletal formulas for TGL and TEA. (b) HSQC spectra of TGL and PbS QDs. The peaks assigned to the $\mathrm{CH}$ groups of TEA and TGL molecules for the PbS QDs are indicated. The different color tone is used to distinguish between odd and even $\mathrm{CH}$ groups.

assign to the $\mathrm{CH}_{3}$ and $\mathrm{CH}_{2}$ groups of the TEA molecule, respectively (see also SI2 in the Supporting Information). These TEA peaks are shifted downfield compared to those in the TEA reference spectrum, thus suggesting a deshielding of the TEA protons in the presence of the QDs. We assign the remaining signals to TGL protons: $2^{\prime}-\mathrm{CH}_{2}(\delta=2.54 \mathrm{ppm}), 2^{\prime \prime}$ $\mathrm{CH}_{2}(\delta=2.65 \mathrm{ppm}), 4^{\prime}-\mathrm{CH}_{2}(\delta=3.52 \mathrm{ppm}), 4^{\prime \prime}-\mathrm{CH}_{2}(\delta=$ 
$3.62 \mathrm{ppm})$, and $3-\mathrm{CH}(\delta=3.717 \mathrm{ppm})$. We note that the chemical shift and line width broadening of these peaks is large compared to those of the free TGL molecules as a consequence of the chemical binding of the TGL molecules to the QD surface. The ${ }^{1} \mathrm{H}-{ }^{13} \mathrm{C}$ heteronuclear single quantum coherence (HSQC) experiments support the assignment of the ${ }^{1} \mathrm{H}$ NMR resonances (Figure 2b). From the HSQC two-dimensional spectrum of the QDs, the signals at $\delta^{\mathrm{C}}=8$ and $46 \mathrm{ppm}$ correspond to the TEA $\mathrm{CH}_{3}$ and $\mathrm{CH}_{2}$ groups, whereas the peaks at $\delta^{\mathrm{C}}=25,75$, and $65 \mathrm{ppm}$ are assigned to the $2-\mathrm{CH}_{2}, 3$ $\mathrm{CH}$, and $4-\mathrm{CH}_{2}$ groups of the TGL molecule, respectively. ${ }^{33}$

The incorporation of $\mathrm{Mn}$ ions into the $\mathrm{QD}$ modifies the ${ }^{1} \mathrm{H}$ NMR spectra (Figure 3). With increasing Mn content up to
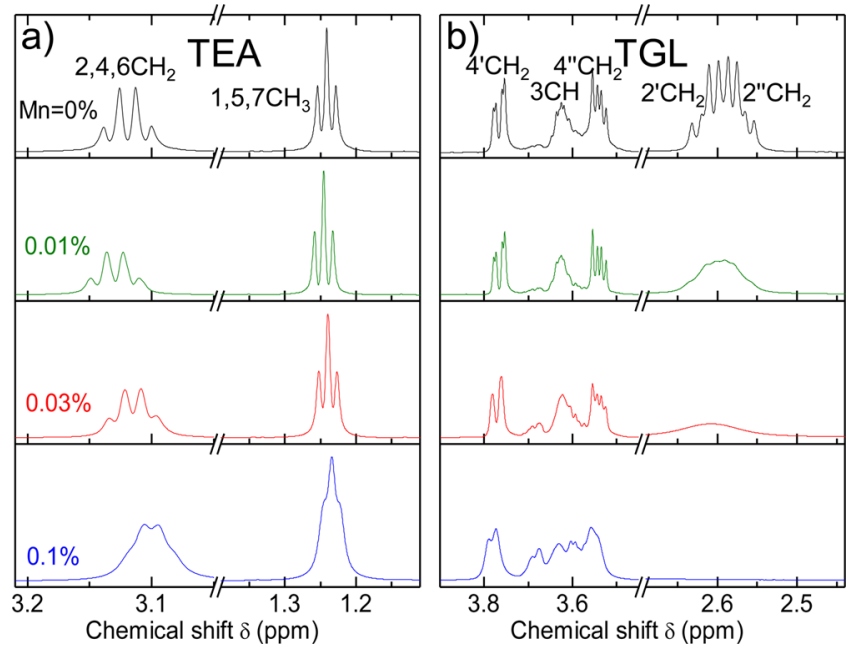

Figure 3. (a) ${ }^{1} \mathrm{H}$ NMR spectra for PbS QDs undoped and doped with Mn concentrations of $0.01 \%, 0.03 \%$, and $0.1 \%$. Peaks corresponding to TEA (a) and TGL (b) $\mathrm{CH}$ groups are indicated.

$0.1 \%$, the characteristic resonance peaks of TEA (Figure 3a) broaden and shift upfield with greater chemical shifts observed for protons on $\mathrm{CH}_{2}-$ groups $(\Delta \delta=-0.08 \mathrm{ppm})$. More significant changes are observed for the resonant peaks of TGL (Figure $3 \mathrm{~b}$ ). All ${ }^{1} \mathrm{H}$ NMR peaks broaden and shift downfield starting from a Mn concentration as low as $0.01 \%$. The most pronounced changes are observed for the $2^{\prime}-\mathrm{CH}_{2}(\delta=2.54$ ppm $)$ and $2^{\prime \prime} \mathrm{CH}_{2}$ groups $(\delta=2.65 \mathrm{ppm})$, where their peaks broaden and their signal becomes undetectable at $\mathrm{Mn}=0.1 \%$. These results suggest that the spin relaxation rates of the protons of the capping ligands and solvent molecules increase due to the magnetic dipolar interactions with the Mn ions. It is worth noting that, for the same Mn concentration, we observe a larger degree of spectral broadening for both the TGL and TEA ${ }^{1} \mathrm{H}$ NMR peaks in a control sample of $\mathrm{MnAc}_{2}$ mixed with free TEA and TGL molecules (see SI3 in the Supporting Information).

From the analysis of ${ }^{1} \mathrm{H}$ NMR spin-lattice relaxation experiments, we estimate the proton spin-lattice relaxation rate constant, $1 / T_{1}$, for each $\mathrm{CH}$ group of TGL (Figure $4 \mathrm{a}$ ) and TEA (Figure $4 \mathrm{~b}$ ) molecules. We observe a general increase of $1 / T_{1}$ with increasing $\mathrm{Mn}$ content. The relaxation rate in TGL molecules increases by up to one hundred times and is different for each $\mathrm{CH}$ group: $1 / T_{1}\left(2 \mathrm{CH}_{2}\right)>1 / T_{1}(3 \mathrm{CH})>1 / T_{1}\left(4 \mathrm{CH}_{2}\right)$. In addition, for each $\mathrm{CH}$ group, $1 / T_{1}$ increases linearly with increasing $\mathrm{Mn}$ content. Instead, the relaxation rate for proton spins of $\mathrm{CH}_{2}$ and $\mathrm{CH}_{3}$ groups in TEA increases by up to ten
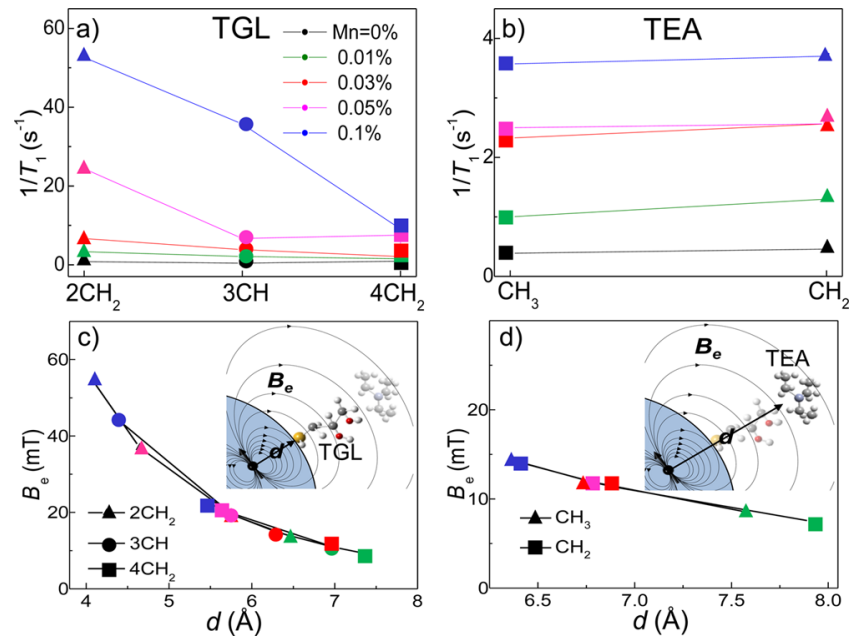

Figure 4. ${ }^{1} \mathrm{H}$ NMR relaxation rates $\left(1 / T_{1}\right)$ for the TGL (a) and TEA (b) $\mathrm{CH}$-groups of $\mathrm{PbS}-\mathrm{Mn}$ QDs with $\mathrm{Mn}$ concentrations: $0 \%, 0.01 \%$, $0.03 \%, 0.05 \%$, and $0.1 \%$. Calculated $\mathrm{Mn}$ induced magnetic field $B_{\mathrm{e}}$ and average distance, $d$ between $\mathrm{Mn}$ and $\mathrm{CH}$ groups of the TGL (c) and TEA (d) molecules in PbS-Mn QDs for different Mn concentrations. Insets in parts $\mathrm{c}$ and $\mathrm{d}$ show the distance $(d)$ between $\mathrm{Mn}$ spins and protons of TGL and TEA molecules and the magnetic field $\left(B_{\mathrm{e}}\right)$ generated by the Mn spin impurity.

times following the $\mathrm{Mn}$ incorporation. The systematic decrease of $1 / T_{1}$ along the $C$ chain of TGL molecules confirms the binding of the TGL to the QD surface at the - $\mathrm{SH}$ site (Figure $4 a)$, while the slower proton $T_{1}$ relaxation for TEA indicates efficient passivation of the QDs with capping ligands, which screen the interactions of the Mn spins with the TEA molecules (Figure 4b).

The proton spin relaxation rate depends on the strength of the magnetic field generated by the paramagnetic impurities, $B_{\mathrm{e}}$, and the distance, $d$, between the paramagnetic impurities and the proton spin. The calculated values of $B_{\mathrm{e}}$ and $d$, as derived from classical magnetic dipolar interactions, and measured values of $1 / T_{1}$ for different $\mathrm{CH}$-groups of TGL and TEA are shown in Figure 4c and d, respectively (see also SI4 in the Supporting Information). For each Mn concentration, the TGL $2 \mathrm{CH}_{2}$-group is always closer to the $\mathrm{Mn}$ ions than $3 \mathrm{CH}$ and $4 \mathrm{CH}_{2}$, respectively (Figure $4 \mathrm{c}$ ). With increasing $\mathrm{Mn}$ content, the average $\mathrm{Mn}-{ }^{1} \mathrm{H}$ distance decreases, while $B_{\mathrm{e}}$ increases for all the inequivalent protons. The calculated distance of $\mathrm{Mn}$ from the $2 \mathrm{CH}_{2}$ group is $d \sim 4 \AA$ for $\mathrm{Mn}=0.1 \%$ and $d \sim 6.5 \AA$ for the lowest concentration $\mathrm{Mn}=0.01 \%$. Since the $\mathrm{S}-2 \mathrm{CH}_{2}$ distance in the TGL molecules is $d \sim 1.5 \AA$, we deduce that the Mn ions are 2.5-5 $\AA$ below the QD surface.

For TEA groups (Figure 4d), we find that, for each $\mathrm{Mn}$ concentration, the $\mathrm{Mn}-{ }^{1} \mathrm{H}$ distance is always larger or comparable to the distance between $\mathrm{Mn}$ spins and TGL $4 \mathrm{CH}_{2}$ groups. For the lowest concentrations (i.e., $\mathrm{Mn}=0.01 \%$ ) the Mn spin sensitivity to distant TEA protons extends up to $d$ $\sim 8 \AA$.

Although ${ }^{1} \mathrm{H}$ NMR is very sensitive to ${ }^{1} \mathrm{H}$-relaxation induced by surrounding electron spins, it only provides an indirect probe of the location and concentration of $\mathrm{Mn}$ ions in the QDs. More generally, the NMR sensitivity is limited by the weak nuclear magnetic moments and fast relaxation times for nuclear species with an electric quadrupole moment making, for instance, ${ }^{55} \mathrm{Mn}$ - and ${ }^{207} \mathrm{~Pb}-\mathrm{NMR}$ transitions undetectable. Pulsed-ESR methods offer the advantage of relying on the 


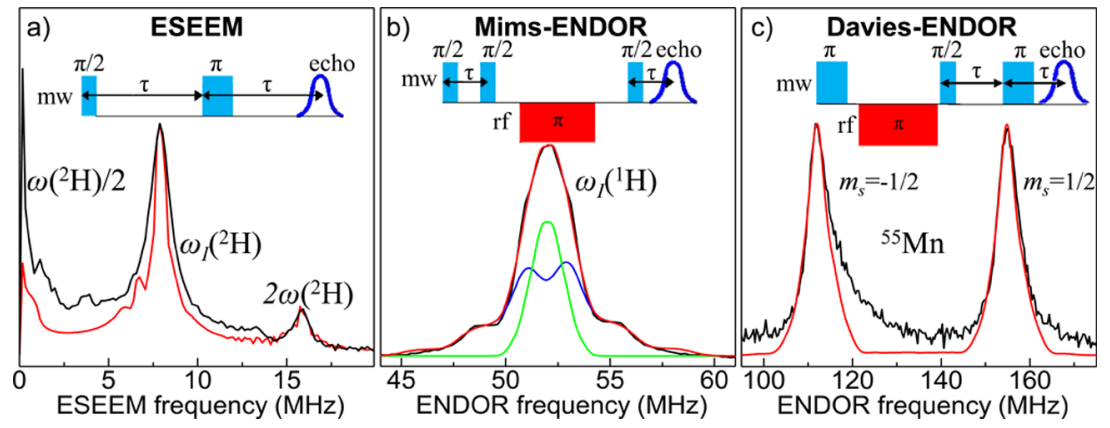

Figure 5. ${ }^{2} \mathrm{H}$-ESEEM (a), ${ }^{1} \mathrm{H}$-ENDOR-Mims (b), and ${ }^{55} \mathrm{Mn}$-Davies-ENDOR (c) spectra at $T=5 \mathrm{~K}$ for the PbS:Mn QD sample along with the simulations (green curve $=$ matrix line, blue curve $={ }^{1} \mathrm{H}$ and ${ }^{2} \mathrm{H}$ interacting with $\mathrm{Mn}$ ions, red curve $=$ total contribution). The ${ }^{1} \mathrm{H}-\mathrm{Mims}$-ENDOR spectrum has been inverted along the intensity axis. Insets: pulse scheme for the ESEEM, Mims-ENDOR, and Davies-ENDOR studies.

detection of electron spin transitions which are much stronger than the nuclear ones because of the larger energy level separation and larger population difference. In pulsed ESR, nuclear spin transitions are detected either by modulation of the electron spin echo signal induced by surrounding nuclear spin fluctuations (i.e., ESEEM) or by saturation of an ESR transition followed by excitation of nuclear spin transitions with radiofrequency signals (i.e., ENDOR). ESEEM and MimsENDOR are more sensitive to low-range nuclear frequencies (typically below $\sim 10 \mathrm{MHz}$ ), whereas Davies-ENDOR is more sensitive in the mid-high frequency range. ${ }^{34}$

The ESEEM spectrum of deuterated Mn-doped PbS QDs $(\mathrm{Mn}=0.03 \%)$ in Figure 5a reveals a resonant peak centered at the Q-band Larmor frequency of deuterium ${ }^{2} \mathrm{H}, \omega\left({ }^{2} \mathrm{H}\right) / 2 \pi=$ 7.9 $\mathrm{MHz}$, combination frequencies $\omega_{\alpha}-\omega_{\beta}$ and $\omega_{\alpha}+\omega_{\beta}$ close to $2 \omega\left({ }^{2} \mathrm{H}\right)$ and $\omega\left({ }^{2} \mathrm{H}\right) / 2$, respectively, as well as several satellite peaks. ${ }^{35}$ We ascribe the ${ }^{2} \mathrm{H}$-ESEEM signal to $\mathrm{Mn}$ spins interacting via dipolar coupling with ${ }^{2} \mathrm{H}$ of the TGL bound to the QDs. ${ }^{36}$ The ${ }^{2} \mathrm{H}$-ESEEM spectrum is simulated by considering a $\mathrm{Mn}$ spin $(S=5 / 2)$ interacting with a ${ }^{2} \mathrm{H}$ nuclear spin $(I=1)$ with hyperfine coupling constants $A_{\text {iso }}=0.16 \mathrm{MHz}$ and $T=0.5 \mathrm{MHz}$ ( $A_{\text {iso }}$ and $T$ are the isotropic and anisotropic contributions to the hyperfine coupling interactions in spherical coordinates, respectively) and an axial nuclear quadrupole constant, i.e., $e^{2} Q q / h \sim 0.3 \mathrm{MHz}$ (see SI5 in the Supporting Information). Similar absolute values for $e^{2} Q q / h$ were previously reported for deuterium. ${ }^{37}$ The larger anisotropic contribution to the hyperfine interaction suggests that the dominant interaction between $\mathrm{Mn}$ and ${ }^{2} \mathrm{H}$ is dipole-dipole. From classical magnetic dipolar calculations, we estimate the $\mathrm{Mn}-{ }^{2} \mathrm{H}$ distances along the principal axis: $d_{x}=d_{y} \sim 12 \AA$ and $d_{z} \sim 6 \AA$ (see SI6 in the Supporting Information).

The ENDOR spectra reveal additional resonances at $\nu=51.5$ $\mathrm{MHz}$ (Figure 5b), 114 and $157 \mathrm{MHz}$ (Figure 5c). The latter two resonances are detected by Davies-ENDOR and are ascribed to Mn electro-nuclear spin transitions within the $m_{\mathrm{s}}=$ $-1 / 2$ and $m_{s}=+1 / 2$ manifolds in the strong coupling regime $\left(2\left|\omega_{I}\right|<|A|\right)$ with the doublet centered at $A / 2$. The obtained value of $A=267 \mathrm{MHz}^{28,38}$ is consistent with the simulation of the continuous wave (CW) ESR spectrum (see SI7 in the Supporting Information). The latter suggests that $\mathrm{Mn}$ ions experience a rhombic symmetry likely due to their proximity to the nanocrystal surface with lower symmetry than the cubic $\mathrm{PbS}$ structure. From first perturbation theory, ${ }^{39}$ the separation $\Delta \nu$ between the Mn nuclear resonance peaks should be twice the Larmor frequency of $\mathrm{Mn}$, i.e., $2 \nu\left({ }^{55} \mathrm{Mn}\right) \sim 25 \mathrm{MHz}$. The larger experimental value of $\Delta \nu \sim 43 \mathrm{MHz}$ is similar to that reported for ${ }^{55} \mathrm{Mn}$ complexes ${ }^{39}$ and ascribed to second-order hyperfine interaction contributions ${ }^{39,40}$ (see SI8 in the Supporting Information). Finally, the peak at $\nu \sim 51.5 \mathrm{MHz}$ resolved by Mims-ENDOR (Figure $5 b$ ) is centered around the natural Larmor frequency of ${ }^{1} \mathrm{H}$ at $B=1.211 \mathrm{~T}$ with doublets symmetrically distributed. We simulate the spectrum by considering a contribution of $\mathrm{Mn}^{2+}$ ions interacting with protons of the TGL bound to the QDs with $A_{\text {iso }}=0.27 \mathrm{MHz}$ and $T=1.3 \mathrm{MHz}$, and a contribution of $\mathrm{Mn}$ ions interacting with residual matrix protons (i.e., TEA and $\mathrm{H}_{2} \mathrm{O}$ ) with $A_{\text {iso }}=0$ $\mathrm{MHz}$ and $\mathrm{T}=0.4 \mathrm{MHz}$. The estimated Mn-TGL ${ }^{1} \mathrm{H}$ distances along the principal axis are $d_{x}=d_{y} \sim 8 \AA, d_{z} \sim 5 \AA$, while for Mn matrix ${ }^{1} \mathrm{H}$, we obtain a lower bound $d_{z} \sim 7 \AA$. These results point toward a compressed octahedral symmetry $\left(d_{z} / d_{x y}=0.6\right)$ whereas, for comparison, the simulated ${ }^{1} \mathrm{H}$-ENDOR spectrum for a control $\mathrm{MnAc}_{2}$ sample mixed with free TGL and TEA molecules reveal a rather symmetric environment $\left(d_{z} / d_{x y}=0.8\right)$ (see SI9 in the Supporting Information).

Overall, both ${ }^{1} \mathrm{H}$ NMR and pulsed-ESR methods enable the observation of electron-nuclear spin interactions at the surface of the QDs and provide us with complementary information on the position and environment of a single paramagnetic center in a nanocrystal. A single $\mathrm{Mn}^{2+}$ impurity in colloidal $\mathrm{PbS}$ QDs experiences a rhombic environment as a result of its proximity to the disordered QD surface. We have estimated the hyperfine dipolar interactions between the $\mathrm{Mn}$ ions and the nuclei of the capping ligands and solvent molecules and their relative distances.

Our findings demonstrate that $\mathrm{Mn}^{2+}$ spins located near the QD surface $(d<5 \AA)$ act as sensors of proton spins located at the $\mathrm{QD}$ surface with $\sim 1 \AA$ sensitivity. Therefore, a minimum amount of $\mathrm{Mn}$ ions (as low as a single ion per QD) is sufficient to induce a fast relaxation of the proton spins of the capping ligands as well as of the solvent molecules: a 60 -fold and 10-fold enhancement for the protons of the capping ligands and solvent molecules, respectively. These features are relevant for the exploitation of $\mathrm{Mn}$-doped colloidal QDs as imaging labels for combined fluorescence and magnetic resonance imaging. We note that at low Mn concentrations the optical properties of QDs are preserved. ${ }^{30}$ Also, for applications of magnetic QDs as electron spin qubits, ${ }^{27,28}$ the interaction of Mn ions with surface protons presents a source of decoherence for electron spins. Our findings indicate that $\mathrm{Mn}-{ }^{1} \mathrm{H}$ dipolar interactions reach a plateau at distances $>6 \AA$, thus providing a guide for developing QD encapsulation techniques to increase the electron spin coherence times. 
More generally, magnetic doping of QDs may pave the way for the use of electron spins as surface sensors in combined pulsed-NMR and ESR studies. Understanding and control of the QD's surface morphology is of great importance for the optimization of the optical and magnetic properties of QDs relevant for the exploitation in optoelectronics ${ }^{3,41}$ and medical imaging, ${ }^{4,30}$ and quantum information processing (QIP). ${ }^{27,28}$

Finally, we envisage that pulsed-ESR could also be sensitive to inequivalent protons ${ }^{42}$ and different $\mathrm{Mn}$ sites, which in our experiments are masked by the line width broadening due to simultaneous excitation of electron-nuclear transitions along different orientations. This sensitivity will provide more information about the distribution of $\mathrm{Mn}$ sites and the distances between a $\mathrm{Mn}^{2+}$ ion and the surrounding inequivalent protons. For instance, these could be achieved by pulsed-ESR experiments either on ordered arrays of QDs or a single QD. Some progress in these directions has recently been achieved by pulsed-ESR experiments on individual atoms on a surface. ${ }^{43}$ Alternatively, more sophisticated pulsed-ESR methods, which greatly increase the resolution of ENDOR spectra, and hence enable us to distinguish between inequivalent nuclear spins, could be implemented. ${ }^{44}$

\section{ASSOCIATED CONTENT}

\section{S Supporting Information}

The Supporting Information is available free of charge on the ACS Publications website at DOI: 10.1021/acs.nanolett.6b02727.

Echo field swept spectrum, detailed explanation of the ${ }^{1} \mathrm{H}$ NMR spectra for TEA and TGL molecules, ${ }^{1} \mathrm{H}$ NMR spectrum of a control sample, $1 / \mathrm{T}_{1}$ relaxation rate formula, description of ESEEM and ENDOR simulations, tables of simulation parameters, dipolar coupling formula and description of CW-ESR simulations (PDF)

\section{AUTHOR INFORMATION}

\section{Corresponding Author}

*E-mail: Fabrizio.Moro@nottingham.ac.uk.

\section{Notes}

The authors declare no competing financial interest.

\section{ACKNOWLEDGMENTS}

This work is supported by The University of Nottingham, The Leverhulme Trust, Grant No. RPG-2013-242, the EPSRC National EPR Facility at The University of Manchester, Grant No. NS/A000014/1, and the EPSRC Integrated Magnetic Resonance Centre for Doctoral Training (Grant No. EP/ J500045/1). We thank Mr C. Howarth for assistance during NMR experiments, Dr. M. W. Fay for TEM images, and Prof. E. J. L. McInnes, Dr. A. Baldansuren, Dr. F. Zamberlan, and Prof. N. R. Thomas for useful discussions.

\section{REFERENCES}

(1) Ray, N.; Staley, N. E.; Grinolds, D. D. W.; Bawendi, M. G.; Kastner, M. A. Nano Lett. 2015, 15 (7), 4401-4405.

(2) Mocatta, D.; Cohen, G.; Schattner, J.; Millo, O.; Rabani, E.; Banin, U. Science 2011, 332 (6025), 77-81.

(3) Saran, R.; Curry, R. J. Nat. Photonics 2016, 10 (2), 81-92.

(4) Jing, L.; Ding, K.; Kershaw, S. V.; Kempson, I. M.; Rogach, A. L.; Gao, M. Adv. Mater. 2014, 26 (37), 6367-6386.

(5) Liu, X.; Yu, M.; Kim, H.; Mameli, M.; Stellacci, F. Nat. Commun. 2012, 3, 1182.
(6) Piveteau, L.; Ong, T.-C.; Rossini, A. J.; Emsley, L.; Coperet, C.; Kovalenko, M. V. J. Am. Chem. Soc. 2015, 137 (43), 13964-71.

(7) Chekhovich, E. A.; Makhonin, M. N.; Tartakovskii, A. I.; Yacoby, A.; Bluhm, H.; Nowack, K. C.; Vandersypen, L. M. K. Nat. Mater. 2013, 12 (6), 494-504.

(8) Morris-Cohen, A. J.; Malicki, M.; Peterson, M. D.; Slavin, J. W. J.; Weiss, E. A. Chem. Mater. 2013, 25 (8), 1155-1165.

(9) Berrettini, M. G.; Braun, G.; Hu, J. G.; Strouse, G. F. J. Am. Chem. Soc. 2004, 126 (22), 7063-7070.

(10) Mikulec, F. V.; Kuno, M.; Bennati, M.; Hall, D. A.; Griffin, R. G.; Bawendi, M. G. J. Am. Chem. Soc. 2000, 122 (11), 2532-2540.

(11) Cure, J.; Coppel, Y.; Dammak, T.; Fazzini, P. F.; Mlayah, A.; Chaudret, B.; Fau, P. Langmuir 2015, 31 (4), 1362-1367.

(12) Gomes, R.; Hassinen, A.; Szczygiel, A.; Zhao, Q.; Vantomme, A.; Martins, J. C.; Hens, Z. J. Phys. Chem. Lett. 2011, 2 (3), 145-152.

(13) Malicki, M.; Knowles, K. E.; Weiss, E. A. Chem. Commun. 2013, 49 (39), 4400-4402.

(14) Harris, R. D.; Amin, V. A.; Lau, B.; Weiss, E. A. ACS Nano 2016, 10 (1), 1395-1403.

(15) Weinberg, D. J.; Dyar, S. M.; Khademi, Z.; Malicki, M.; Marder, S. R.; Wasielewski, M. R.; Weiss, E. A. J. Am. Chem. Soc. 2014, 136 (41), 14513-14518.

(16) Knowles, K. E.; Malicki, M.; Parameswaran, R.; Cass, L. C.; Weiss, E. A. J. Am. Chem. Soc. 2013, 135 (19), 7264-7271.

(17) Makinen, M. W.; Mustafi, D.; Kasa, S. ENDOR of Spin Labels for Structure Determination: from Small Molecules to Enzyme Reaction Intermediates. In Biol. Magn. Reson., Berliner, L. J., Ed.; Kluwer Academic Publisher: OH, 2002; Vol. 14.

(18) Borbat, P. P.; Costa-Filho, A. J.; Earle, K. A.; Moscicki, J. K.; Freed, J. H. Science 2001, 291 (5502), 266-269.

(19) Jeschke, G.; Polyhach, Y. Phys. Chem. Chem. Phys. 2007, 9 (16), 1895-1910.

(20) Baranov, P. G.; Orlinskii, S. B.; Donega, C. D.; Schmidt, J. Phys. Status Solidi B 2013, 250 (10), 2137-2140.

(21) Baranov, P. G.; Orlinskii, S. B.; Donega, C. d. M.; Schmidt, J. Appl. Magn. Reson. 2010, 39 (1-2), 151-183.

(22) Yavkin, B. V.; Mamin, G. V.; Orlinskii, S. B.; Gafurov, M. R.; Salakhov, M. K.; Biktagirov, T. B.; Klimashina, E. S.; Putlayev, V. I.; Tretyakov, Y. D.; Silkin, N. I. Phys. Chem. Chem. Phys. 2012, 14 (7), 2246-2249.

(23) Gafurov, M.; Biktagirov, T.; Mamin, G.; Klimashina, E.; Putlayev, V.; Kuznetsova, L.; Orlinskii, S. Phys. Chem. Chem. Phys. 2015, 17 (31), 20331-20337.

(24) Barnard, A. S. Analyst 2009, 134 (9), 1751-1764.

(25) Jelezko, F.; Wrachtrup, J. Phys. Status Solidi A 2006, 203 (13), 3207-3225.

(26) Schirhagl, R.; Chang, K.; Loretz, M.; Degen, C. L., NitrogenVacancy Centers in Diamond: Nanoscale Sensors for Physics and Biology. In Annu. Rev. Phys. Chem., Johnson, M. A.; Martinez, T. J., Eds. 2014; Vol. 65, pp 83-105.10.1146/annurev-physchem-040513103659

(27) Ochsenbein, S. T.; Gamelin, D. R. Nat. Nanotechnol. 2011, 6 (2), 111-114.

(28) Moro, F.; Turyanska, L.; Wilman, J.; Fielding, A. J.; Fay, M. W.; Granwehr, J.; Patane, A. Sci. Rep. 2015, 5, 10855.

(29) Turyanska, L.; Moro, F.; Knott, A. N.; Fay, M. W.; Bradshaw, T. D.; Patanè, A. Part. Part. Syst. Char. 2013, 30 (11), 945-949.

(30) Turyanska, L.; Hill, R. J. A.; Makarovsky, O.; Moro, F.; Knott, A. N.; Larkin, O. J.; Patanè, A.; Meaney, A.; Christianen, P. C. M.; Fay, M. W.; Curry, R. J. Nanoscale 2014, 6 (15), 8919-25.

(31) Moro, F.; Turyanska, L.; Granwehr, J.; Patanè, A. Phys. Rev. B: Condens. Matter Mater. Phys. 2014, 90 (20), 205428.

(32) Stoll, S.; Schweiger, A. J. Magn. Reson. 2006, 178 (1), 42-55.

(33) We note that the composition of the solvents, i.e., the ratio of $\mathrm{H}_{2} \mathrm{O}$ to $\mathrm{D}_{2} \mathrm{O}$, has no effect on the chemical shift and line broadening observed in the QD samples.

(34) Schweiger, A.; Jeschke, G. Principles of pulse electron paramagnetic resonance; Oxford University Press, 2005. 
(35) We note that the contribution of ${ }^{1} \mathrm{H}$ is not observed in the ESEEM because the modulation depth of nuclear modulations scales with the factor $I(I+1)$ in absence of nuclear quadrupole coupling. Given that $I=1 / 2$ for ${ }^{1} \mathrm{H}$, the ${ }^{1} \mathrm{H}$ modulation depth is $8 / 3$ times smaller than ${ }^{2} \mathrm{H}$.

(36) The combination peak $\omega_{\alpha}-\omega_{\beta}$ appears as a narrow feature because orientation-dependent hyperfine interactions are partially refocussed, therefore revealing the orientation dependent contribution to the ESEEM spectral broadening and line shape.

(37) Dikanov, S. A.; Tsvetkov, Y. Electron Spin Echo Envelope Modulation (ESEEM) Spectroscopy; CRC Press: 1992.

(38) Abragam, A.; Bleaney, B. Electron paramagnetic resonance of transition ions; Oxford University Press: Oxford, 1970.

(39) Sturgeon, B. E.; Ball, J. A.; Randall, D. W.; Britt, R. D. J. Phys. Chem. 1994, 98 (49), 12871-12883.

(40) Boettcher, R.; Poeppl, A.; Lorenz, M.; Friedlaender, S.; Spemann, D.; Grundmann, M. J. Magn. Reson. 2014, 245, 79-86.

(41) Turyanska, L.; Makarovsky, O.; Svatek, S. A.; Beton, P. H.; Mellor, C. J.; Patane, A.; Eaves, L.; Thomas, N. R.; Fay, M. W.; Marsden, A. J.; Wilson, N. R. Adv. Electron. Mater. 2015, 1 (7), 1500062 .

(42) Murray, A. T.; Dowley, M. J. H.; Pradaux-Caggiano, F.; Baldansuren, A.; Fielding, A. J.; Tuna, F.; Hendon, C. H.; Walsh, A.; Lloyd-Jones, G. C.; John, M. P.; Carbery, D. R. Angew. Chem., Int. Ed. 2015, 54 (31), 8997-9000.

(43) Baumann, S.; Paul, W.; Choi, T.; Lutz, C. P.; Ardavan, A.; Heinrich, A. J. Science 2015, 350 (6259), 417-420.

(44) Jeschke, G.; Schweiger, A. J. Magn. Reson., Ser. A 1996, 119 (1), $45-52$. 